\title{
TV/Series
}

18 | 2020

Séries et espace

Topographies fantastiques dans la fiction télévisée francophone : Les Revenants (Canal +, $2012 ; 2015)$ et Zone blanche (France 2, 2017-2019)

\section{Audrey Evrard}

\section{OpenEdition}

Journals

Édition électronique

URL : http://journals.openedition.org/tvseries/4718

DOI : $10.4000 /$ tvseries.4718

ISSN : 2266-0909

Éditeur

GRIC - Groupe de recherche Identités et Cultures

Référence électronique

Audrey Evrard, «Topographies fantastiques dans la fiction télévisée francophone : Les Revenants (Canal +, 2012 ; 2015) et Zone blanche (France 2, 2017-2019)», TV/Series [En ligne], 18 | 2020, mis en ligne le 15 septembre 2020, consulté le 08 décembre 2020. URL : http://journals.openedition.org/ tvseries/4718; DOI : https://doi.org/10.4000/tvseries.4718

Ce document a été généré automatiquement le 8 décembre 2020.

\section{(i) $९$

TV/Series est mis à disposition selon les termes de la licence Creative Commons Attribution - Pas d'Utilisation Commerciale - Pas de Modification 4.0 International. 


\title{
Topographies fantastiques dans la fiction télévisée francophone : Les Revenants (Canal +, 2012 ; 2015) et Zone blanche (France 2, 2017-2019)
}

\author{
Audrey Evrard
}

1 Jugée bien souvent trop provinciale, peu sophistiquée, difficile à exporter, pour ne pas dire médiocre, la série télévisée française reste peu étudiée. Et pourtant, le phénomène sériel fascine, le public certes, mais le milieu universitaire également, pour preuve les nombreux articles, ouvrages et revues publiés en France sur le sujet $\cdot s_{i}$ ces publications scannent le vaste paysage de la série américaine depuis plus de dix ans, lintérêt n'est pas aussi enthousiaste pour la production francophone. Depuis quelques années, cependant, cette dernière cherche à s'installer malgré la domination toujours très forte des États-Unis, concurrencés malgré tout par la Grande-Bretagne et les pays scandinaves depuis le début des années 2000. Deux séries francophones relativement récentes, Les revenants (Canal +, 2012; 2015) et Zone Blanche (France 2, 2017-2019), seront donc l'objet de cet article.

2 C'est à la petite maison de production indépendante Haut et Court que l'on doit Les Revenants, projet initialement conçu en réponse à une commande de Canal +. Fondée en 1992 avec l'objectif de renouveler la production cinématographique française et de l'accompagner à l'international, Haut et Court a étendu son champ d'action à la télévision dès 2008, ce qui les a menés à développer plusieurs collaborations avec ARTE, Canal + et quelques autres partenaires européens. Premier gros succès international de cette maison de production, Les Revenants a rapidement attiré l'attention de distributeurs américains, dont Sundance Channel, ce qui lui a valu de remporter l'Emmy pour la meilleure série dramatique internationale en 2013. Une fois la deuxième saison sortie, c'est sur Netflix que les spectateurs américains ont pu redécouvrir cette série, l'original ayant reçu un meilleur accueil que le remake, The Returned, financé par la chaîne A\&E (2015), annulé après la diffusion de quelques épisodes. La genèse de cette adaptation télévisée du long-métrage Les Revenants (2004), 
réalisé par Robin Campillo et produit par Haut et Court, explique la qualité cinématographique de cette série. Céline Sciamma et Emmanuel Carrère sont intervenus à différentes étapes de la conception du scénario, qui a finalement été repris par Fabrice Gobert dont le premier long-métrage, Simon Werner a disparu, venait d'être nommé pour le César du meilleur premier film en 2011.

Production France Télévision, Zone blanche illustre l'amorce d'une nouvelle ligne éditoriale plus ambitieuse pour la chaîne publique depuis quelques années. Celle-ci a notamment motivé la mise en œuvre de projets tels que Les Témoins (co-production franco-belge, France 2/Channel 4/BBC Four/La Une, 2014-2017), Le Chalet (2018) et La Forêt (co-production franco-belge, France 3/La Une). Les droits de diffusion de ces trois séries ont d'ailleurs été achetés par Netflix, qui les a ajouté à son catalogue nordaméricain en 2018. Récompensée à plusieurs festivals avant même la diffusion de la première saison, Zone blanche est née de l'imagination du scénariste Mathieu Missoffe, déjà connu pour ses talents dans le genre policier. ${ }^{1}$ Cet article a été développé avant la diffusion de la deuxième saison aux États-Unis, c'est pourquoi je me concentrerai exclusivement sur la première saison dans l'analyse proposée dans les pages qui suivent. ${ }^{2}$

4 Très différentes dans leurs intrigues, Les Revenants et Zone blanche présentent néanmoins plusieurs points communs. Elles partagent un réseau intertextuel et référentiel riche, largement inspiré de séries américaines cultes, notamment Twin Peaks (David Lynch, 1990-91 ; 2017), et de la vague scandi-noir qui déferle sur les petits écrans européens et nord-américains depuis 2010. Ces influences jouent pour beaucoup dans l'investissement finalement assez rare en France dans le genre fantastique. Les Revenants propose ainsi une incarnation réaliste du "zombie », personnage qui, bien qu'ayant ses origines dans la culture vaudou haïtienne et caribéenne, s'est installé dans l'imaginaire cinématographique américain à la fin des années 1960 suite au succès des films de George Romero, notamment Night of the Living Dead (1968). Les morts-vivants des Revenants ne ressemblent, cependant, en rien aux corps en état de décomposition avancée que nous avons l'habitude de voir dans les fictions américaines, telles que The Walking Dead (AMC, 2010-present), The Fear of the Walking Dead (AMC, 2015-present), ou plus récemment Game of Thrones (HBO, 2011-2019). Zone blanche apporte, quant à elle, des inflexions nouvelles à la série policière, inspirée du " polar ", genre bien établi dans la tradition française et très prisé par les spectateurs nationaux. Cette série explore ainsi de nouvelles frontières dans cet univers du polar, empruntant au fantastique, au western et aux mythologies celtes et gallo-romaines. Chacune de ces séries s'inscrit ainsi dans un espace spécifique, qui (1) contribue pleinement au(x) genre(s) exploré(s), (2) reprend une géographie et une topographie complexes qui font écho à celles déployées dans le scandi-noir, et (3) dont la construction souligne l'émergence d'interrogations écologiques nouvelles tout en encourageant une réflexion critique sur la place de la fiction française dans une topographie internationale redessinée et en perpétuel mouvement.

5 En 2009, dans son article «Lost in Globalized Space? A Certain French Cinema Abroad ", Roger Célestin posait la question de la place du cinéma français dans un espace culturel désormais global(isé) ${ }^{3}$. Les efforts, et succès relatifs, d'une production télévisuelle plus volontairement francophone que strictement française depuis quelques années justifient de rediriger cette interrogation du cinéma vers la télévision. Ce questionnement de la place de la série télévisée francophone dans le monde est 
d'autant plus pertinent si l'on considère l'enjeu que dessinait alors Célestin, à savoir : «France's ability to consistently produce and export commercially successful films that are neither 'Hollywoodian' nor (...) films that recycle a 'certain idea of France' ${ }^{4}$ ». Les efforts de renouvellement et d'internationalisation de la série télévisée francophone engagés depuis plusieurs années (1) ne cherchent plus à se démarquer de la production internationale, notamment américaine, mais semblent vouloir (2) questionner et subvertir cet enfermement de la production culturelle française dans les dichotomies soulignées ci-dessus.

6 Cet article examine donc la mise en scène de plus en plus commune dans les séries télévisées policières et/ou fantastiques francophones (principalement françaises et franco-belges) de territorialités composites et topographies mobiles. M'appuyant principalement sur Les Revenants (Fabrice Gobert, 2012, 2015) et Zone blanche (2017), je me concentre sur ce qui m'apparait comme une double impulsion paradoxale dans le travail des scénaristes et réalisateurs francophones ces dernières années. Inspirées par l'engouement international suscité par l'esthétique sérielle scandinave et l'accent que celle-ci met sur une temporalité longue et plus lente de la progression de l'intrigue et de sa résolution, ces séries opèrent, d'une part, une déterritorialisation visible du récit. En effet, bien que celui-ci se déroule systématiquement dans une communauté rurale ou du moins étrangement isolée, l'espace, assemblage d'éléments hétéroclites et marqueurs géographiques divers, échappe à toute localisation spécifique et se définit plutôt par son caractère dénationalisé. D'autre part, et c'est là qu'émerge le paradoxe, ces séries revendiquent la centralité narrative et esthétique d'un enracinement géographique et écologique double. Affirmant un lien inextricable entre l'humain et le naturel, chaque série mobilise des récits mythologiques très spécifiques à l'histoire locale. Alors que tout effort de différencier espaces local, national et global semble volontairement déstabilisé, le paysage y est néanmoins réactivé et animé, dépassant les fonctions strictement spectaculaires et identitaires qui lui étaient traditionnellement assignées. Les univers que nous y découvrons dans leurs diverses manifestations atmosphériques, écologiques et géologiques, se transforment en sites/lieux définis autant par leur immobilisme que par leur mobilité. Ils acquièrent alors, comme le proposait Gilles Deleuze dans son livre L'Image-Temps (1985), la capacité de signifier à la fois l'absence et la présence, le vide et le plein, la vie et la mort. Si l'intertextualité référentielle de ces séries suggère une appropriation, et une transposition, de codes génériques étrangers, je propose que nous y voyions plutôt la mise en scène et le questionnement de ce rapport à deux niveaux: (1) tel qu'il structure le récit et les thématiques adressées, mais aussi (2) tel qu'il détermine l'économie de la production et de l'esthétique télévisuelles francophones contemporaines.

\section{Espaces composites - Délocalités}

7 Les Revenants commence avec le retour de Camille, une adolescente, disparue quatre ans plus tôt, avec sa classe, dans un tragique accident de bus sur cette route de montagne sinueuse. Véritable choc pour sa famille, ce retour est le premier d'autres à venir. Plusieurs « revenants » apparaissent ainsi au cours de cette première saison: Simon, Serge, Madame Costa, et Victor. Mort trente-cinq ans plus tôt, Victor, jeune garçon mystérieux, vit en fait caché, depuis toutes ces années, auprès de son père. Isabelle Rachel Casta note le fait que tous ces revenants sont décédés de morts violentes: 
meurtres, accidents, suicides ${ }^{5}$. Leur présence suscite diverses émotions au sein de la communauté des vivants: soulagement, méfiance et rejets violents. La tension est d'autant plus forte que ces retours ravivent des traumatismes personnels et collectifs et déstabilise le quotidien tranquille des habitants de la ville. La première saison se termine sur le repli des vivants à La Main Tendue, refuge tenu par Pierre, alors que « la horde » des revenants, toujours plus nombreux, se prépare à quitter les lieux, avec, parmi elle, Simon, Camille, Madame Costa, Victor, ainsi que la mère de Camille et Julie, une jeune femme qui avait pris Victor sous son aile, forcé de sortir de sa cachette suite à l'hospitalisation de son père.

8 La deuxième saison reprend six mois plus tard dans un paysage dévasté : une grande partie de la ville est désormais inondée suite à la montée soudaine des eaux du réservoir. La région est en état d'urgence ; une forte présence militaire est venue prêter main forte aux gendarmes locaux. Adèle est enceinte de Simon, inquiète quant à la nature de son enfant à naître ; Jérôme, le père de Camille, et Léna, sa sœur jumelle, surmontent chacun à leur façon le départ de Camille et de sa mère. Un nouveau venu, Berg, apparaît: c'est le fils de l'ingénieur qui, plus de trente ans auparavant, avait dessiné les plans du barrage. Cette deuxième saison opère un va-et-vient entre ces deux mondes, celui des revenants retranchés dans un lotissement épargné par les eaux, mais abandonné, et celui des vivants refugiés dans quelques quartiers et lieux de la ville ayant été épargnés par les inondations. Berg et Jérôme s'associent dans leurs efforts de trouver un passage vers l'autre côté, le premier dans l'espoir de retrouver son père, et le second dans le but de ramener sa famille. La série se conclut sur plusieurs images: les revenants se jetant dans un gouffre, Victor/Louis aux côtés de Julie, tous les deux " vivants ", sur une plage, et l'enfant d'Adèle et Simon abandonné sur le pas de la porte d'un pavillon.

Le film de Campillo avait pour cadre une agglomération de province, de taille moyenne, confrontée, comme le reste du monde, au retour massif et inexpliqué de personnes décédées parmi les vivants. La série resserre l'intrigue, s'installant dans une petite ville «rurbaine » accrochée à la montagne, en plein cœur des Alpes et bornée par un immense barrage semi-circulaire. Initialement développée pour durer deux saisons, Les Revenants s'installe dans un environnement assez difficile à qualifier. Aucune information plus précise n'est en effet donnée aux spectateurs afin qu'ils puissent mieux géolocaliser l'intrigue. Il n'est d'ailleurs jamais fait mention d'un au-delà géographique, cette petite ville semblant exister dans un isolement étrange, indépendamment de toute société extérieure. Mais ce qui frappe les spectateurs dès les premiers épisodes de la saison une, c'est surtout l'hybridité architecturale et topographique de ce décor. Si les chalets accrochés à la montagne ainsi que les fermages isolés ont leur place dans ce petit village alpin, d'autres plans révèlent des lieux plus représentatifs d'un environnement péri-urbain, telles les barres d'immeubles où habite Julie ou encore les vues aériennes nocturnes qui suggèrent une étendue urbaine plongée dans le noir par des pannes de secteur inexpliquées. La présence de bâtiments incongrus, marqueurs d'un ailleurs renforce le caractère disparate de cette localité impossible à situer, aussi utopique que dystopique. Un diner américain, "America Diner ", servant burgers, milk-shakes et frites, trône aux marges de la petite ville, surmonté d'une enseigne inattendue dans ces lieux (Exemple : Fig. 1). Un peu plus loin, le Lake Pub est le bar excentré où les jeunes viennent se retrouver, boire et jouer au billard, et où se croisent plusieurs personnages récurrents. Si ces marqueurs créent 
un effet de distance pour les spectateurs français, ils dé-francisent, par la même occasion, le paysage pour un public international, permettant ainsi au récit de se relocaliser un peu partout.

Fig. 1. Les Revenants (S. 1, Ep. 5, 2015)

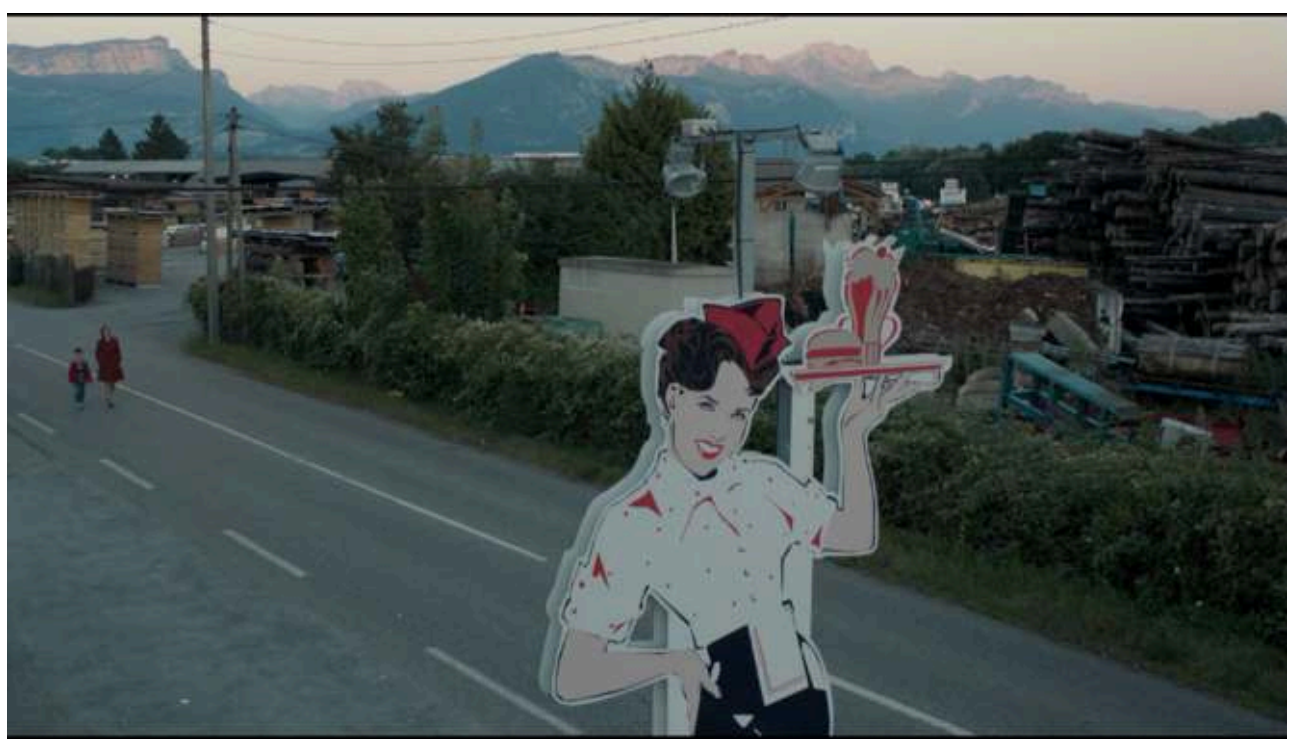

Pour le critique Josué Morel, peu convaincu par la série lors de sa sortie en 2012, la visibilité et l'intrusion de ces marqueurs étrangers démontrent l'incapacité de cette série de se démarquer, justement, de ses modèles américains ${ }^{6}$. Cette dépendance évidente vis-à-vis les codes et conventions en vigueur Outre-Atlantique se retrouve, selon lui, dans l'écriture-même du récit, plus particulièrement dans sa tonalité religieuse (signalée par la confiance qu'Adèle a dans le prêtre du village, et la centralité de La Main Tendue, un lieu où se réfugieront tous les habitants pour la confrontation finale avec les revenants dans le dernier épisode de la première saison) ou encore l'évolution sécuritaire de l'intrigue (la poursuite d'un tueur en série dominant la saison une, et la crise militaire et humanitaire de la saison deux). Pour Morel, cette hybridité géographique sape l'originalité de cette série et la possibilité de redéfinir la série française sur de nouvelles bases nationales. Au lieu de cela, Les Revenants ne fait que reproduire, assez mal selon lui, ses modèles américains.

11 Si la série Les Revenants revendique une parenté avec des genres et des récits américains, la construction composite de l'espace mis en scène est, à mes yeux, plus complexe. L'indétermination, voir la non-détermination volontaire, de ce lieu sert bien évidemment les intérêts d'une série alliant réalisme et surnaturel. Offrant une variation sur un genre typiquement américain, Les Revenants s'éloigne du Far West (post)-apocalyptique de The Walking Dead (AMC, 2010-present) and Fear of the Walking Dead (AMC, 2015-present), deux séries ayant pour décors respectifs le Sud des EtatsUnis, dans l'état de Géorgie, et Los Angeles. Ces dernières épousent les grands espaces et orchestrent des transhumances massives de morts-vivants et des vivants qui cherchent à leur échapper dans l'horizontalité de la géographie nord-américaine. Les Revenants creuse, en contraste, la verticalité de son récit, de son territoire et d'une temporalité où passé, présent et futur se confondent de plus en plus à mesure que nous avançons vers la conclusion. 
Si la dislocation architecturale et culturelle de la ville et de la communauté présentées dans Les Revenants leur permet de s'inscrire dans un espace global, elles n'en sont pas moins détachées et dissociées des cadres régional et national qu'elles semblent pourtant déborder. Casta observe ainsi que le principe esthétique primordial de la série est la circularité :

Tout se passe comme si les contraintes par le cadre (un barrage, c'est ce qui «barre »! Mais aussi les montagnes escarpées, l'eau menaçante...) s'exprimaient toujours sur le mode de l'outre-passement, du débordement, de la fuite et du tropplein ${ }^{7}$.

Bien qu'évoluant dans un espace clos, borné et «barré », les personnages de la série tracent, par le biais de déambulations continues, une cartographie interne et interstitielle. Seuls deux personnages entrent et sortent, à l'exception des revenants : Laure, la petite-amie de Julie, officier de la gendarmerie locale, disparait entre les deux saisons, sans explication; Berg, quant à lui, entre dans ce monde clos au début de la deuxième saison. Ce sont les déplacements de Léna, Lucy, Camille, Simon, Serge, et d'autres, à travers ce micro-territoire composite qui y révèlent tout un réseau de tunnels, routes, et passages reliant les diverses zones et composantes de ce paysage impossible à concevoir dans son intégralité. S'il est possible de circuler à l'intérieur de ce petit monde, toute tentative d'en sortir entraîne les personnages dans une circularité infinie, sans issue. C'est ce que réalisent Julie, Laure et Victor à la fin de l'épisode 7 de la saison une : tinté de rouge, le passage de ce dernier tunnel qui devait les conduire au-delà du barrage les redirige au contraire dans une boucle infernale qui se termine sur ce barrage infranchissable (Fig. 2). Les deux femmes se regardent, inquiètes, dans un long silence qui conclut cet épisode.

Fig. 2. Le tunnel menant au barrage. Les Revenants (S. 1, Ep. 7)

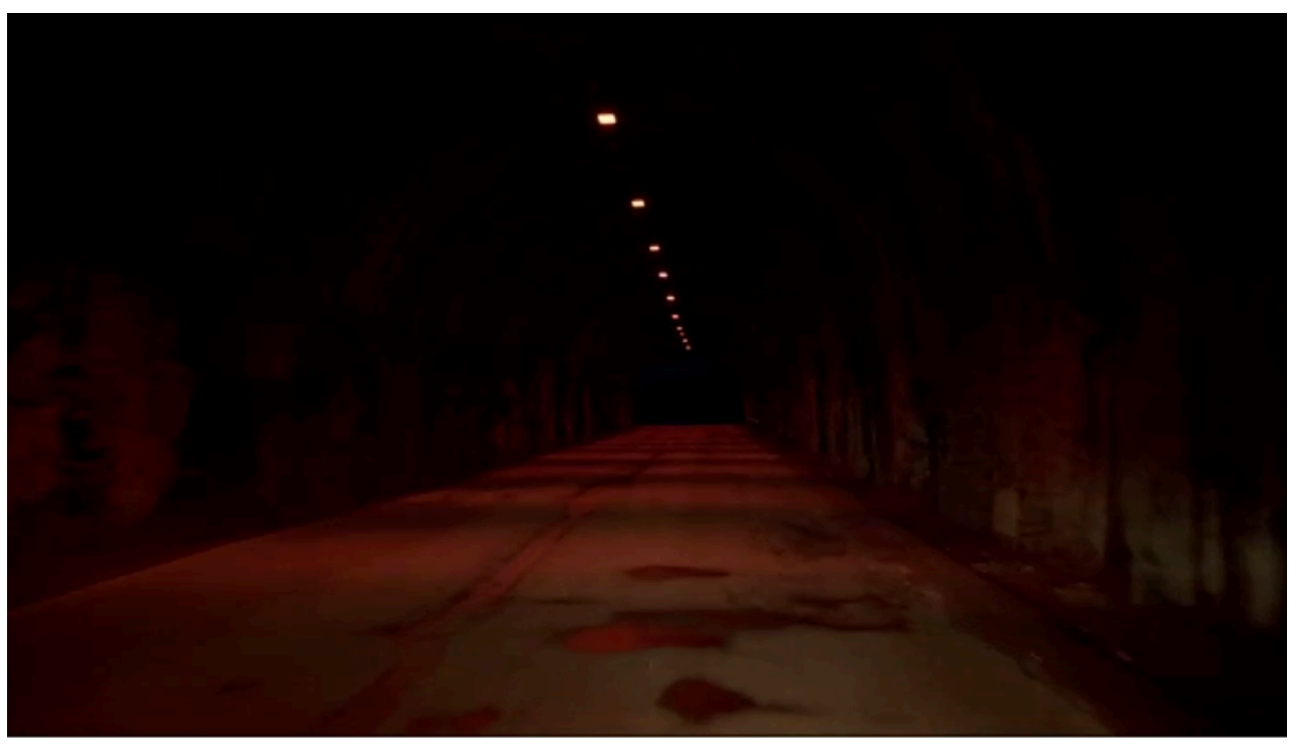

Ce réseau creuse néanmoins au cœur de cet enclos plusieurs mobilités qui le transforment en une territorialité affective qui s'inscrit dans un espace-temps refermé sur lui-même. Aux passages physiques, matérialisés par la cinématographie, s'en superposent d'autres, surréels et fantasmés. L'espace qui se construit, et se reconstruit constamment, sous nos yeux regorge ainsi de ponts, tunnels, portes, couloirs, etc. De la même manière, la césure qui sépare les deux saisons introduit un effet de miroirs et de 
distorsions entre deux rendus du même monde. Dans cette deuxième saison, la topographie de ce territoire apparaît comme radicalement différente, complètement redessinée: de nouveaux paysages sont apparus, d'autres ont disparu, de nouveaux sites se sont ouverts alors que d'autres ont été engloutis, sortis de notre angle de vision. Ces mutations continues minent les efforts répétés des spectateurs de saisir la totalité de cette géographie éclatée et lacunaire. Ce n'est que dans la deuxième saison, par l'intermédiaire de Jérôme, le père de Camille, qui ne peut se résoudre à abandonner sa femme et sa fille, et de Berg que nous pouvons commencer à nous représenter plus formellement cette topographie mobile.

Dans les six mois qui séparent les événements présentées dans les deux saisons, Jérôme a développé une carte (Fig. 3) et identifié une spatialité régie par un système de portes et de sas (Fig. 4). Comme il l'explique à Léna et à Berg, ces points de liaisons ne peuvent être activés que par l'alignement d'une géographie immobile organisant l'espace en surface et d'une topographie mobile structurant les strates souterraines de ce territoire. Si cette carte reste mystérieuse et ne répond pas à toutes les questions que nous pose le récit, on peut néanmoins assimiler cette démarche, et la fonction endossée par Jérôme, à celle d'un topographe qui souffrirait de ce que Robert Tally a plus récemment qualifié de "topophrénie » - "a condition of 'disease' [...]. Topophrenia characterizes the subjective engagement with a given place, with one's sense of place, and with the possible projection of alternative spaces $^{8} »$. Ces tensions, et la reconceptualisation de l'espace qu'elles génèrent, qualifient parfaitement l'obsession folle, selon Léna, de Jérôme qui le pousse à vivre comme un reclus depuis le départ des revenants six mois plus tôt. La clé de l'énigme soulevée par Les Revenants, et à laquelle la fin de la série ne nous donne pas vraiment de solution satisfaisante, ne serait-elle pas justement à chercher et à trouver dans cette cartographie « topophrénique »?

Fig. 3. La carte de Jérôme. Les Revenants (S. 2, Ep. 4)

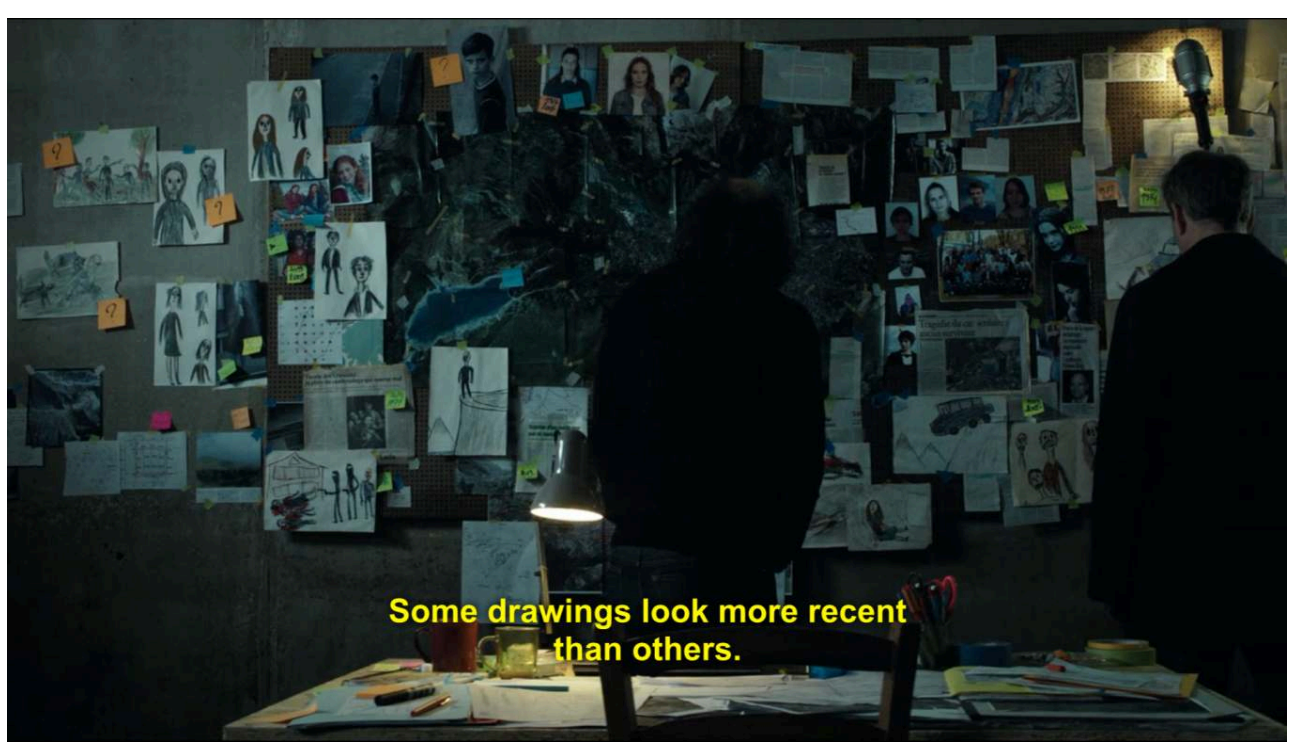


Fig. 4. Jérôme explique le système de portes et de sas qu'il pense avoir découvert. Les Revenants (S. 2, Ep. 4)

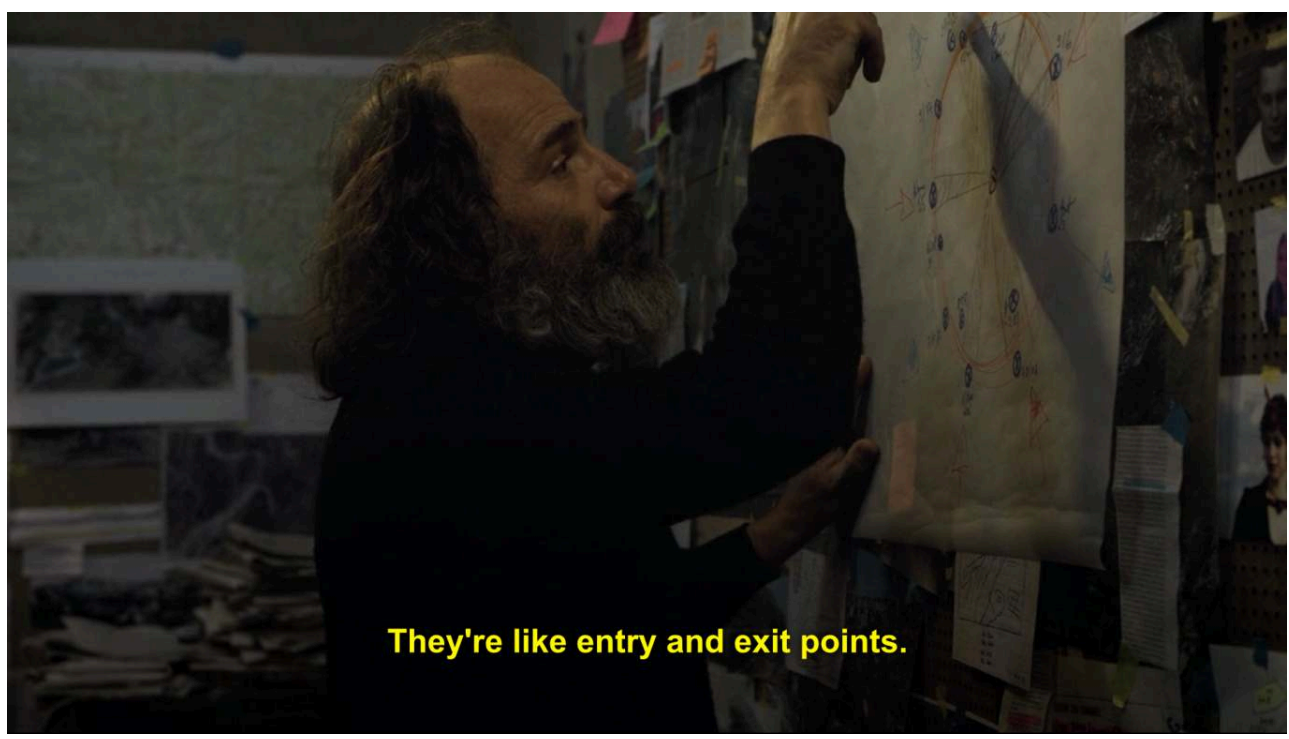

La construction de l'espace dans Zone blanche est en plusieurs points similaire à celle des Revenants. Tout aussi fantastique, étrange et déstabilisant, le monde insulaire imaginé par Mathieu Missoffe s'appuie sur les conventions du polar, un genre beaucoup plus familier pour le public français. La zone blanche, imperméable aux ondes électromagnétiques, c'est la petite ville de Villefranche, et l'immense forêt qui la jouxte. Missoffe a choisi le nom de Villefranche pour deux raisons principales: premièrement, c'est le nom de sa ville de naissance, et qu'il avoue souffrir d'allergies causées par le milieu rural ; deuxièmement, il existe un grand nombre de Villefranche en France. Cette identification est donc à la fois très déterminée par sa ruralité et extrêmement générique du fait de la prolifération diffuse de cette appellation sur tout le territoire national. Dans Les Revenants, c'est le barrage qui apporte la singularité ultra-locale, comme l'ont très bien analysé Catherine E. Clark et Brian Jacobson'. Dans Zone blanche, c'est le nom choisi par le groupe d'éco-guerriers, Les enfants d'Arduinnia, qui la signale. Arduinna est le nom d'une déesse gallo-romaine, vénérée dans ce qui est aujourd'hui la vaste région limitrophe des Ardennes qui s'étend sur trois pays, la France, la Belgique et le Luxembourg.

17 Au début de la première saison, nous arrivons donc à Villefranche avec le procureur Siriani, bien décidé à comprendre pourquoi «le taux d'homicides [y] est six fois supérieur à la moyenne nationale » (9'04-S01E01). Extrêmement dramatique, virant au grotesque, cette première scène affirme d'entrée le paysage référentiel dont se sont inspirés Missoffe et ses réalisateurs, à savoir Twin Peaks. Seul, sur une route déserte, le corps de Siriani succombe et rampe tant bien que peu vers une voiture, au beau milieu de la présence écrasante de cette nature menaçante, baignée dans une brume épaisse le tout au son de la chanson, diégétique, "Mister Lonely ", écrite en 1962 par l'auteurcompositeur Bobby Vinton, alors qu'il combattait au Vietnam. Les paroles traduisent l'émotion d'un soldat, envoyé au front, loin de sa famille, et privé de tout moyen de communication. Dans la série, ce soldat pourrait être cet homme que nous voyons, dans ces premières secondes, survivre de peu à la piqûre d'un insecte, après être sorti de sa voiture pour localiser un point de connexion pour son téléphone portable. La séquence qui précède le générique se concentre un court instant sur le panneau signalant au 
voyageur sa bienvenue à Villefranche, clin d'œil à celui que l'agent Cooper croise du regard à son arrivée à Twin Peaks en 1990.

Quelques minutes plus tard, Siriani fera la rencontre du Commandant de gendarmerie Laurène Weiss, enfant du pays, mère célibataire et rescapée, vingt ans plus tôt, d'un enlèvement particulièrement troublant. Tenue captive pendant plusieurs jours au cœur de la forêt, son petit ami de l'époque, le maire actuel de la ville, l'avait retrouvée laissée pour morte, avec deux doigts en moins. Depuis, Weiss garde un lien obsessionnel avec cette forêt. Plusieurs enquêtes rythment cette première saison, et beaucoup ont pour cadre cette immense étendue végétale. Alors que chaque épisode voit la découverte, l'investigation et la résolution d'un meurtre local sordide, plusieurs récits se développent sur l'ensemble des huit épisodes. C'est en partant à la recherche de sa meilleure amie, la fille du maire, disparue depuis six mois, que Cora, la fille de Weiss, se rapproche des Enfants d'Arduinna. Cette deuxième intrigue éco-politique se poursuit sur la deuxième saison. En cela, Zone blanche se conforme à la bifurcation désormais typique du récit sériel promue par la production scandinave, et repris depuis par les scénaristes américains, dans des séries comme True Detective, (HBO, 2014-2019) ou Forbrydelsen, (DR1, 2007-2012), adapté aux Etats-Unis sous le titre (The Killing, AMC, 2011-2013; Netflix, 2014), pour en citer quelques exemples. Le Commandant de gendarmerie Weiss, que nous ne voyons jamais en uniforme, rappelle d'ailleurs beaucoup l'inspectrice Sarah Linden, héroïne de The Killing (2011-2014). Comme elle, Laurène Weiss arpente les bois, souvent seule, de jour ou de nuit, sous un rideau de pluie ou dans le brouillard, y recherchant des indices pour ses enquêtes ou tout simplement pour satisfaire son rapport obsessionnel, quasi fusionnel, avec cette forêt.

L'hybridité territoriale de Villefranche se confirme rapidement, soutenue autant dans l'économie visuelle de la série que dans la bande-son. Comme dans Les Revenants, le cœur social de la petite ville, très éclatée géographiquement, est un bar, l'Eld_rado (il semble manquer un néon, plongeant le premier $\mathrm{O}$ du nom dans le noir), tenue par Sabine, interprétée par Brigitte Sy. La musique country y rythme les conversations ; bikers et ouvriers de la scierie locale y croisent gendarmes et représentants politiques locaux. On se croirait plus dans un bar en plein cœur du Midwest américain que dans les Vosges ou la forêt des Ardennes. Le son du banjo qui structure la musique du générique et ponctue les épisodes nous transporte dans les paysages reculés des Appalaches, ce que les deux compositeurs de la série, Thomas Couzinier et Frédéric Kooshmanian, confirment avoir voulu suggérer ${ }^{10}$. Le banjo introduit, selon eux, une dimension plus végétale et intemporelle. Kooshmanian explique ainsi que «les cordes du banjo [...] faisaient penser à l'écorce des arbres, à la nature ", élément fondamental à la facture narrative, visuelle et dramatique de la série. Ainsi, si Fabrice Gobert a souhaité inscrire Les Revenants à la rencontre de diverses conceptions du fantastique, l'une très américaine et l'autre beaucoup plus française, Mathieu Missoffe, créateur de Zone Blanche, revendique un jeu de genres plus éclectique, mêlant polar, fantastique et western. Pour contrarier un peu plus l'univers hypermasculin de ces genres, Missoffe choisit de concentrer la série sur Weiss, un personnage féminin, farouche, complexe et solitaire. Cette optique situe Zone blanche dans un corpus transnational de fictions policières récentes comprenant, entre autres, The Fall (BBC2, 2013-2016), Top of the Lake (BBC Two, 2013-présent) et Marcella (ITV, 2016-2018). Toutes ont pour sujet principal une femme, victime, dans le passé, de traumatismes violents qui se trouve forcée de les 
revivre à travers ses enquêtes qui la confrontent à diverses découvertes macabres et à un ou plusieurs tueurs en série s'acharnant sur le corps d'autres femmes ${ }^{11}$.

Les termes géographiques et cartographiques par lesquels Mathieu Missoffe, créateur de Zone Blanche, résume la progression dramatique et scénaristique qui nous conduit de la première à la deuxième saison rappellent les principes examinés plus tôt dans Les Revenants :

L'enjeu était de faire coïncider la quête géographique de Laurène avec les secrets de la ville, ce qui était beaucoup plus tortueux sur la première saison. J'avais envie de plus de codes d'aventure, donc moins de polar - forcément, l'un compensant l'autre, et ce motif de la carte, qui est toujours important. Maintenant, c'est le moment de remplir les blancs sur cette carte, ce qui est toujours très important : on a fini notre exposition, on a brossé notre univers, et on peut jouer avec tous ces outils ${ }^{12}$.

21 Le motif de la carte apparaît donc plus visiblement dans la deuxième saison, comme c'était le cas dans Les Revenants. Malgré son référentiel américain (musique, intertextes), Zone Blanche emprunte également beaucoup à la territorialité télévisuelle britannique et scandinave. La série reprend ainsi à son compte les principes esthétiques, philosophiques et moraux qui occupent, selon Glen Creeber, une place centrale dans les fictions criminelles scandinaves ${ }^{13}$. On y retrouve ainsi l'affirmation visuelle d'une esthétique très sombre, accentuant les bleus, les verts, et les ombres, déjà typique du film noir, une atmosphère renforcée d'autre part par le grand nombre de scènes nocturnes, crépusculaires ou filmées au petit matin (Exemple, Fig. 5).

Fig. 5. Vue aérienne d'ensemble de la courte séquence générique. Zone blanche (S. 1, Ep. 7, 2017)

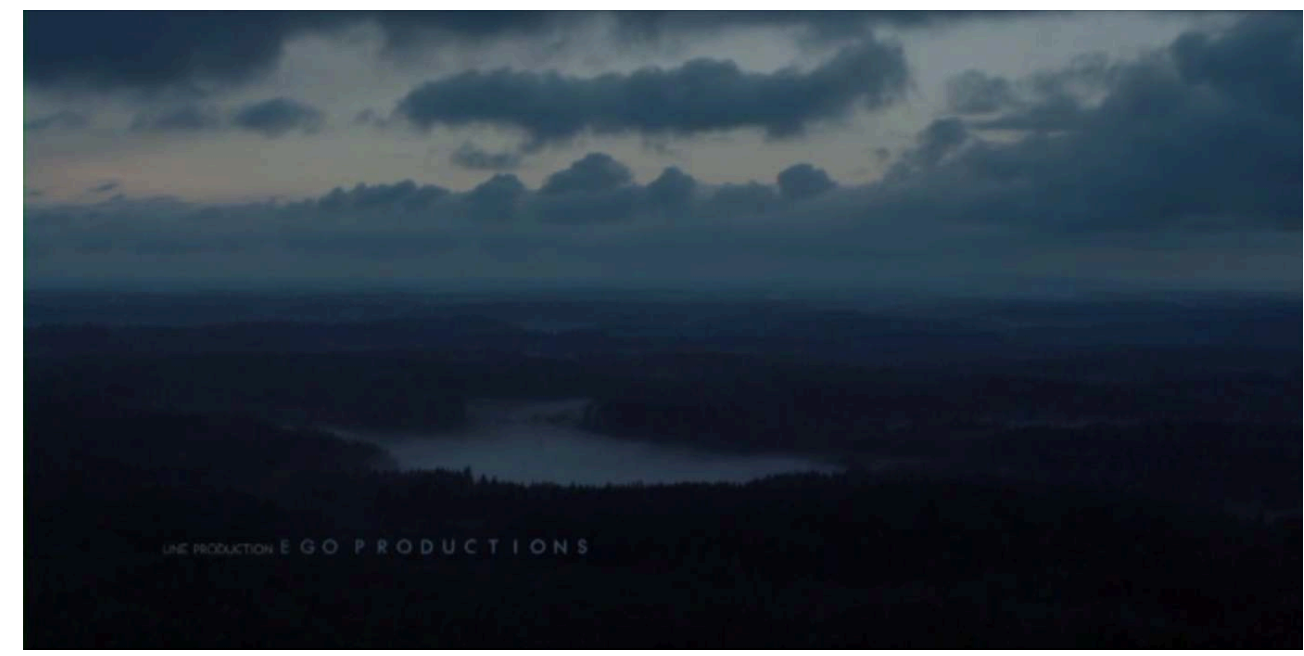

Comme dans la fiction scandinave, Zone Blanche développe une intrigue plurielle, se déployant sur plusieurs plans, conférant à la série un rythme différencié. Illustration directe du constat fait par le procureur à son arrivée concernant le taux d'homicides exceptionnel de la région, chaque épisode se concentre sur l'élucidation d'un meurtre ; ces enquêtes sont l'occasion pour les créateurs de la série de déployer le milieu social de Villefranche, rongé par les non-dits, les rituels sordides, et la corruption morale. L'enlèvement de la fille du maire occupe l'ensemble de la saison, et n'est résolue qu'au huitième et dernier épisode de la série. Un troisième niveau développe l'intrigue écologique, opposant les propriétaires de la scierie locale aux Enfants d'Arduinna. Cette intrigue se poursuit sur l'ensemble de la saison 2, tout comme l'obsession du 
Commandant Laurène Weiss de retrouver ce(lui) qui l'a kidnappée vingt ans plus tôt et gardée captive au fond d'une grotte dans la forêt pendant plusieurs jours. Ces deux intrigues explorent des thématiques mythologiques, politiques, économiques, et écologiques, qui ont pour ancrage la forêt, comme réalité physique, végétale, et symbolique. Dans cet entrelacs, le territoire naturel acquiert une double valeur, comme le précisait Gunhild Agger dans son étude de The Killing. Topographie concrète, il prend une dimension plus symbolique et universelle à travers l'accent mis sur ses qualités atmosphériques ${ }^{14}$. La seule différence étant que, dans Zone blanche, l'univers urbain laisse place à un territoire rural où la nature n'est ni tout à fait bienveillante ni totalement hostile.

Dans les différents entretiens accordés aussi bien par Fabrice Gobert que par Mathieu Missoffe, les deux réalisateurs n'expriment aucun complexe à s'inspirer d'une dramaturgie de genres bien établis dans la production internationale. Leurs propos soulignent, bien au contraire, la volonté de «jouer » et de déstabiliser ces codes et ces schémas. Cette démarche reprend ainsi un trait assez spécifique au cinéma d'auteur français hérité de la Nouvelle Vague, visible notamment dans le travail de cinéastes tels qu'olivier Assayas et Claire Denis.

Dans la deuxième partie de cet article, je me tourne plus précisément sur la construction spatio-temporelle des univers fantastiques que nous rencontrons dans Les Revenants et Zone blanche. Fabrice Gobert opte pour une esthétique qui mêle le merveilleux et l'étrange au familier, le fantastique à l'ordinaire. Conjurant une tradition française limitée, mais néanmoins populaire, celui-ci travaille dans cette série une esthétique de l'entre-deux, où des phénomènes surnaturels et inexplicables viennent déranger un quotidien apparemment ordinaire et tranquille. De la même manière, le fantastique dans Zone blanche naît du mystère qui plane autour de cette forêt, et des phénomènes étranges dont le personnage principal, Weiss, est témoin. Mathieu Misoffe se revendique plus explicitement, cependant, « des films fantastiques qui mettent en scène la révolte de la nature ${ }^{15}$. " Si l'impératif de reconnaissance du monde représenté est crucial dans le réalisme fantastique à la française, Fabrice Gobert, Mathieu Missoffe, Julien Despaux et Thierry Poiraud (les deux co-réalisateurs de Zone blanche) s'efforcent de déstabiliser les limites locales et nationales de ce processus. Ainsi, Les Revenants et Zone Blanche ne cherchent pas tant, selon moi, à se conformer aux normes établies par les studios américains. Il s'agit plutôt pour ces créateurs de mettre en scène l'inertie dans laquelle la création télévisuelle francophone se trouve aujourd'hui du fait de la prédominance d'une production qui se veut dépositaire d'un imaginaire national tout autant qu'elle aspire à étendre sa visibilité à l'international. Ces séries seraient donc à la fois mises en abyme de cette inertie et mises en œuvre créatives de s'en extraire. A cette fin, le local devient un point d'ancrage depuis lequel il devient possible d'engager certaines angoisses générées par les élans centripètes d'un imaginaire de plus en plus globalisé, et leurs ravages sociologiques, culturels et écologiques.

\section{Topographies « originaires »}

Reconsidérer le caractère composite délibéré de ces deux séries à la lumière de Gilles Deleuze et du concept d'espace quelconque qu'il emprunte à Pascal Auger permet de creuser plus profondément la dimension symbolique de l'espace dans ces deux séries. 
Dans L'Image-Mouvement (1983), Deleuze reprend ainsi ce terme à son propre compte et en propose cette définition :

Un espace quelconque n'est pas un espace universel abstrait, en tout temps, en tout lieu. C'est un espace parfaitement singulier, qui a seulement perdu son homogénéité, c'est-à-dire le principe de ses rapports métriques ou la connexion de ses propres parties, si bien que les raccordements peuvent se faire d'une infinité de façons. C'est un espace de conjonction virtuelle, saisi comme pur lieu du possible. Ce que manifestent en effet l'instabilité, l'hétérogénéité, l'absence de liaison d'un tel espace, c'est une richesse en potentiels ou singularités qui sont comme les conditions préalables à toute actualisation, à toute détermination [...] L'espace quelconque serait l'élément génétique de l'image-affection ${ }^{16}$.

Comme je l'ai démontré dans la première partie, les deux localités mises en scène dans Les Revenants et Zone blanche affirment leur singularité géographique et topographique locale alors même que chaque série semble reposer sur la quête narrative du lien et de la cohérence. Non seulement ce décrochage offre au fantastique un point d'appui parfait, mais la géographie interstitielle qui se matérialise en conséquence permet aux créateurs de ces séries de sortir des sentiers battus de la production télévisée nationale. Cette tension ouvre plusieurs possibilités de liaisons entre des imaginaires nationaux différents mais contigus - comme le nord de la France et la Belgique par exemple - ou entre des géographies éparses confrontées à une même angoisse du vide vers/dans lequel la globalisation nous happe.

Dans Les Revenants, le monde que Gobert construit visuellement semble constamment tendu entre deux coordonnées opposées, la vie et la mort, le mouvement et l'inertie. Cependant, dans ce monde, la vie ne présuppose pas nécessairement le mouvement, tout comme mort et inertie ne vont pas nécessairement de soi non plus. Cette tension apparaît dès la séquence générique, créée par le graphiste Batmanu. Dans un entretien, celui-ci explique avoir voulu capturer dans le même temps la vie et la mort, tirant profit d'un effet visuel, le «tilt-shift, qui permet de dire la mise au point sur un seul élément de l'image, le reste étant flou, pour voiler les rapports de tailles ${ }^{17}$. » Les plans fixes qui se succèdent sur la durée de cette séquence parviennent ainsi à communiquer deux états incompatibles. Nous déplaçant du sommet de la montagne vers le village, au creux de la vallée, les images choisies par Batmanu préservent, du même coup, l'intégrité géographique de ce microcosme. En effet, cette séquence, qui met l'accent sur les paysages, resitue les silhouettes humaines des revenants dans un paysage qui semble avoir été vidé de toute vie humaine. Le thème musical, composé par le groupe écossais Mogwai, accentue une temporalité paradoxale, impossible, prise entre précipitation et suspension, à laquelle font échos le glissement rapide des nuages et les mouvements à peine perceptibles des sujets animés présents.

La mise en scène s'organise plus largement dans les épisodes autour d'une opposition qui évoque celle que Deleuze a établi entre espaces vides et natures mortes dans les premières pages de L'image-temps (1985). Pour Deleuze, la nature morte « ne se confond pas avec un paysage ", elle "se définit par la présence et la composition d'objets qui s'enveloppent en eux-mêmes, » elle " est le temps ", et par là, pleine ${ }^{18}$. L'espace vide, en contraste, "vaut avant tout par l'absence d'un contenu possible "; "intérieurs ou extérieurs, (ils) constituent des situations purement optiques (et sonores) ${ }^{19}$. Il est $^{2}$ intéressant de noter que la nature morte se fait beaucoup plus fortement ressentir dans la composition visuelle des plans de la première saison, alors que les spectateurs se 
retrouvent confrontés à des espaces désormais vides, dans la deuxième saison, suite à la montée des eaux qui a fait fuir les habitants.

Fig. 6. Victor suit Julie avant leur première rencontre. Les Revenants (S. 1, Ep. 1, 2012)

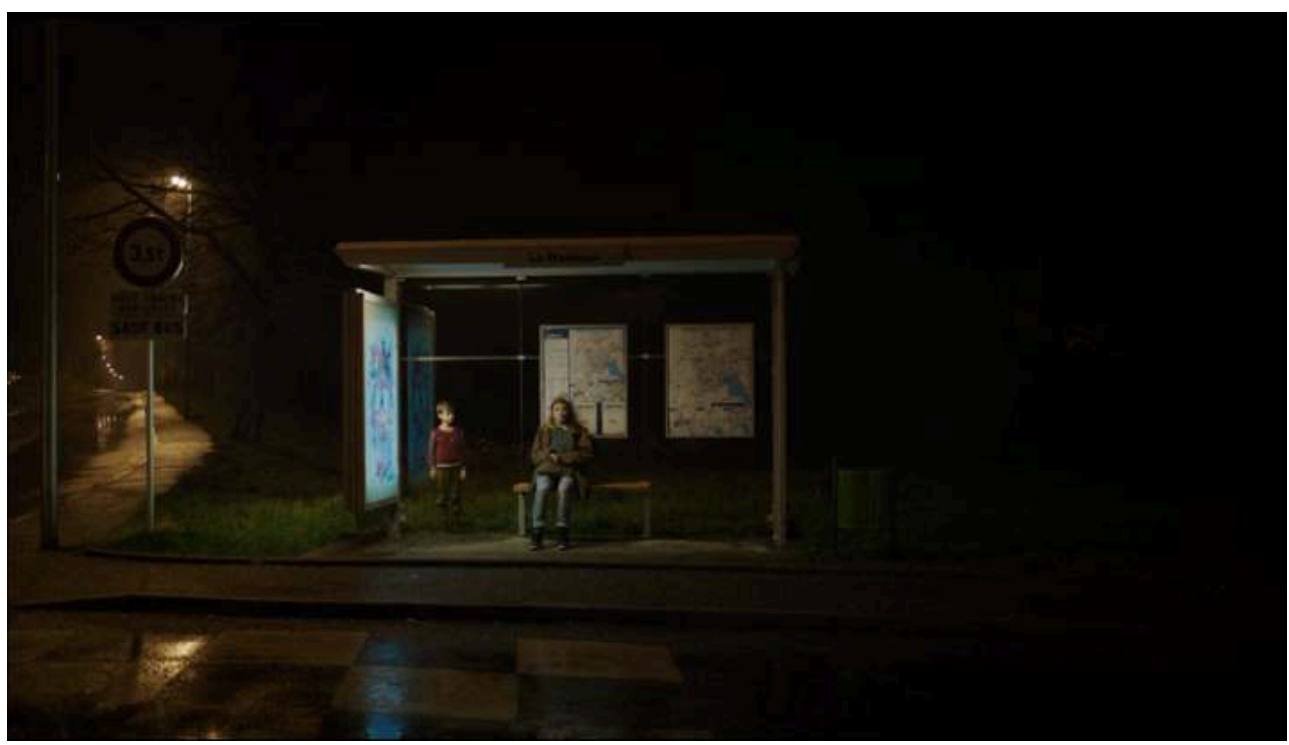

Dans l'expérience visuelle du changement, la nature morte nous donne ainsi accès au travail du temps, lui conférant une tangibilité qui, autrement, nous échappe.

L'influence de l'univers du photographe américain Gregory Crewdson sur la mise en scène et la cinématographie des Revenants renforce cette dichotomie entre natures mortes et espaces vides. Tant dans le choix des plans, que dans la palette des couleurs déployées, Gobert transpose l'esthétique du photographe à l'écran. Celle-ci appuie la qualité déconcertante de la banlieue américaine, en s'intéressant à ce qui dans son quotidien évoque simultanément le banal et l'extraordinaire. L'imbrication de cadres dans le cadre, l'immobilité des sujets humains dans cet environnement tinté de bleus, verts et jaunes se retrouvent dans de nombreux plans et scènes de la série (Exemple Fig. 6). Pour Julien Foulatier, galeriste familier avec le travail de Crewdson, la singularité de ses photographies tient au fait que chacun de ses clichés nous amène à la limite du monde que nous regardons. Au moment où nous le regardons, ce monde est sur le point de subir un renversement dramatique, de basculer dans l'obscure. Les mots qu'il utilise pour décrire les sentiments ressentis devant les clichés de Crewdson pourraient s'appliquer, de manière troublante, au monde que nous observons dans Les Revenants :

Peut-être verrez-vous dans ces personnages livides des sortes de revenants ou, au contraire, des êtres bien vivants momifiés par un quotidien trop pesant ou un secret trop lourd à porter. Peut-être verrez-vous dans ces scènes ce qu'elles ont de plus banal ou, au contraire, ce qu'elles ont de plus paranormal. En tout cas, tout semble basculer ${ }^{20}$.

31 Dans Les Revenants, les choix esthétiques brouillent continuellement la frontière qui sépare ce que nous percevons comme vivant et ce que nous présumons être déjà mort. Les personnages vivants (Adèle et Julie, entre autres), nous apparaissent régulièrement plus pâles, plus transparents que les revenants eux-mêmes, particulièrement dans la première saison. La saison deux introduit des signes distinctifs cependant, notamment la dégradation physique qui commence à affecter le corps de plus en plus de revenants. 
Les premiers signes apparaissent dès la saison une, affectant paradoxalement Lena, la sœur jumelle, vivante, de Camille. La peau de son dos révèle une infection cutanée inexpliquée et inexplicable, semblable à celles qui surgiront sur le corps d'autres revenants dans la saison suivante.

La récurrence de surfaces (miroirs, fenêtres, eau) permet des effets de transparence et de superposition entre les vivants et les revenants, et donc les morts (Exemples : Fig. 7-9). Ces effets visuels suggèrent un processus d'embaumement de ces personnages dans leur environnement surréel. Le méticuleux travail de composition de chaque plan inscrit Les Revenants dans une tension non résolue, mais fondamentale au succès du récit. Ces effets de transparence offrent une mise en abyme du rapport extrêmement ténu, mais aussi paradoxal, que l'esthétique des Revenants construit entre immobilisme et mouvement.

Fig. 7-9. Les Revenants (S01E06, 2012 ; S01E01, 2012)
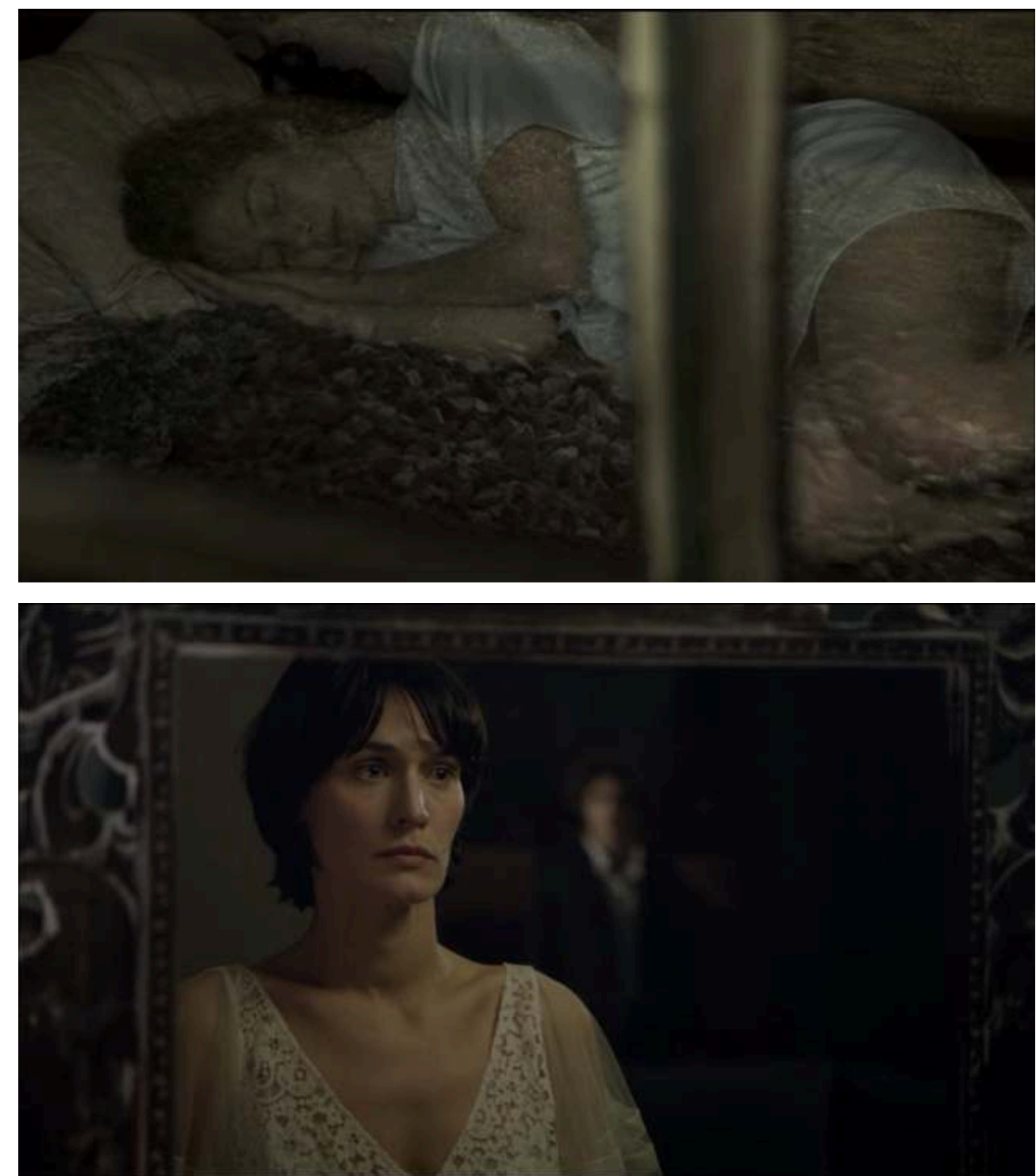


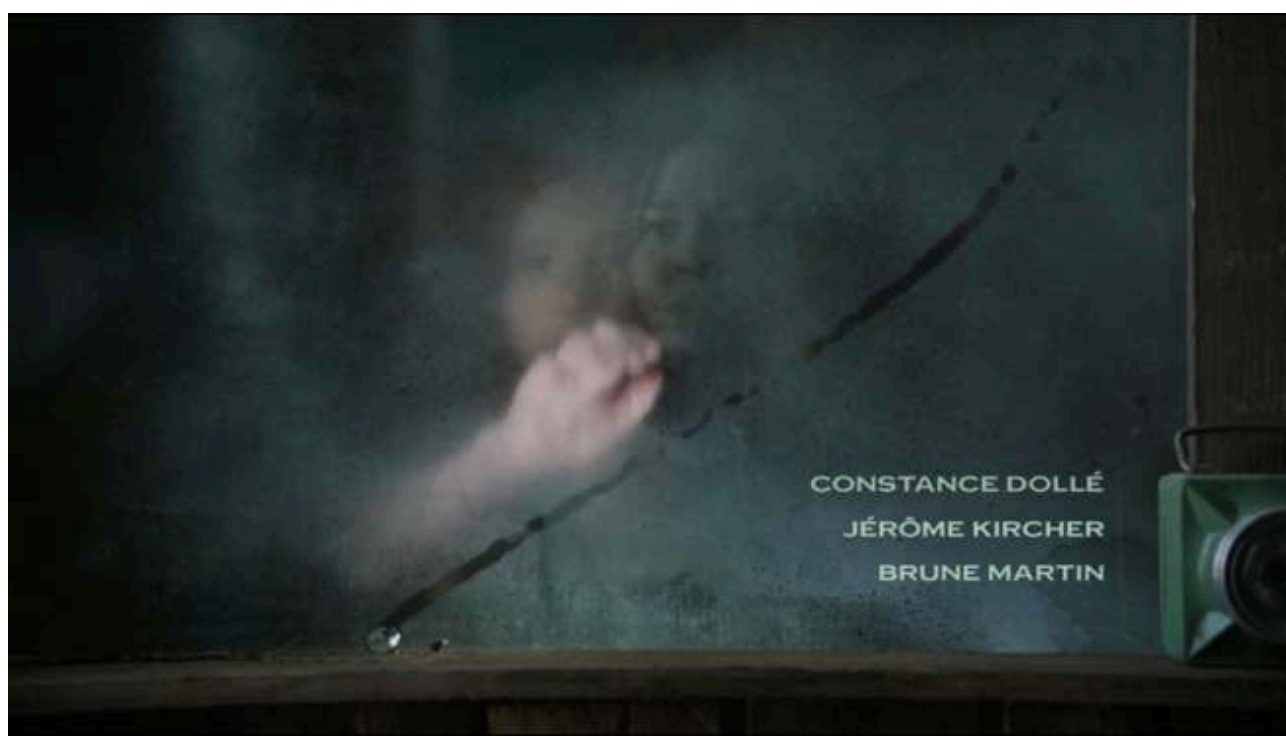

Dans son livre, Le cinéma de l'immobilité (2007), Ludovic Cortade examine la corrélation qui peut exister entre $\mathrm{im} /$ mobilité ou immobilisme et la crédulité des spectateurs devant le récit filmique.

La privation de mouvement n'implique pas systématiquement la suspension de la croyance du spectateur, mais peut au contraire s'avérer être un élément constitutif de celle-ci. Certes, l'immobilisation de l'image au cinéma peut rompre l'illusion, relevée par Christian Metz, de la "présence réelle du mouvement", gage de la croyance du spectateur. Tel est en particulier le cas lorsque l'arrêt sur image apparait comme le signe d'un arrêt final de la narration, effectuant ainsi un retour abrupt du spectateur à la réalité de ce qui n'était qu'une succession d'images projetées sur un écran. Pourtant, l'analyse a également permis de dégager une mobilisation des ressources esthétiques de l'immobilité non pour suspendre la croyance du spectateur, mais au contraire pour l'intensifier : le figement, le repos, l'apatheïa et l'inertie participant ainsi à une esthétique de la transparence ${ }^{21}$.

On peut tracer dans l'esthétique fantastique déployée dans Les Revenants la présence d'une mise en relation similaire, et d'une esthétique de la transparence qui emprunte à la définition proposée par Cortade. Cette transparence intervient aussi bien dans le motif du reflet, récurrent dans la série, qu'au niveau de la structuration du récit. Plusieurs effets de miroir ponctuent notre expérience : premièrement, les vivants et les revenants sont continuellement reliés par un fil tenu; deuxièmement, les deux saisons sont séparées par une césure ; troisièmement, l'espace du récit s'organise à travers un dédoublement territorial - la géographie de surface et les diverses strates qui dessinent une topographie et une géologie souterraines. Ces duplications différenciées peuvent également être mises en relation, à un niveau encore plus large, excédant le cadre narratif de la série, entre, d'un côté, la crise écologique mise en scène dans le récit et, de l'autre côté, celle à laquelle nous sommes confrontés avec de plus en plus d'urgence. Quant à l'inertie inhérente au récit, elle n'est pas sans rappeler celle paralysant la création télévisuelle française.

Pour Ludovic Cortade, l'inertie se définit comme l'incapacité «à opérer une jointure entre la permanence du mouvement et un sens qui lui serait immanent ${ }^{22} »$. En nous laissant contempler la répétition d'un cycle auquel nous venons d'assister - Lucy dépose l'enfant d'Adèle et Simon, enfant d'une vivante et d'un revenant, devant la porte d'étrangers - la conclusion de la série affirme ce principe d'inertie comme 
moteur et résolution de la série. S'agirait-il ici finalement de l'énigme centrale posée par cette série ? Si c'est le cas, le monde microcosmique que nous avons exploré se transforme sous nos yeux en une vaste nature morte, une image-temps ou une mémoiremonde qui ne cesserait de se remplir et de se reconstituer. Toute variation, tout mouvement et tout changement perceptible dans ce monde n'auraient alors pour fonction que de nous rendre sensible à ce processus, attirant notre attention sur «la perspective du temps ${ }^{23}$ ».

La tension que Deleuze voit entre la nature morte et l'espace vide sous-tend la collusion du fantastique et du réalisme qui définit l'esthétique des Revenants, mais aussi celle de Zone Blanche. Dans ces deux séries, les paysages naturels débordent et excèdent la spectacularité cinématographique qui les a traditionnellement définis. Ainsi, si les plans aériens larges invitent à pénétrer le substrat affectif et atmosphérique de ces paysages (Exemple, Fig. 5), les derniers épisodes de la première saison de Zone blanche mettent en œuvre une prise de contact beaucoup plus haptique de la forêt, nous plongeant littéralement dans l'humus (Exemples, Fig. 10-11). Cette intimité libère le substrat mythologique, fusion de traditions celtes et gallo-romaines, qui structure plus explicitement le récit dans la deuxième saison. Les dernières images du dernier épisode de la première saison nous laissent entrevoir la silhouette de cette figure mythique, Cernunnos, divinité celte du monde animal, de la vie et de la fertilité, clairement identifiée, qui, dans la deuxième saison, s'affirme comme une force ambigüe, destructrice mais avec laquelle Laurène entretient un lien singulier (Exemple, Fig. 10). Dans la foulée de cette créature surnaturelle, l'épisode se conclut sur une plongée verticale révélant le corps nu de Laurène Weiss recouvert de matériaux végétaux. Dans ce dernier plan, Laurène sort de son état inconscient, dans un souffle de torpeur (Exemple, Fig. 11). Cette dernière image semble nous révéler Laurène comme l'incarnation de la déesse Arduinna, communément représentée accompagnée d'un ours (le surnom "Nounours» de son co-équipier prend alors un nouveau sens) et vénérée pour ses vertus protectrices vis-à-vis la Forêt des Ardennes et la faune locale.

Fig. 10-11. Zone blanche (S. 1, Ep. 8, 2017)

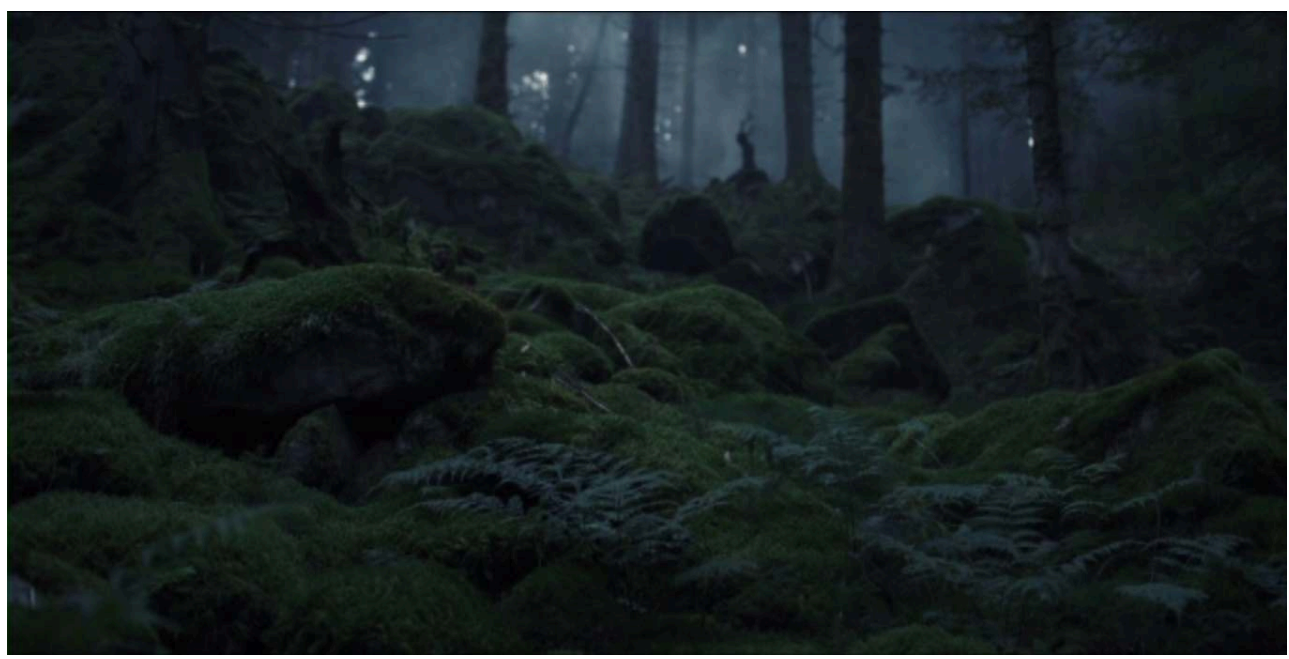




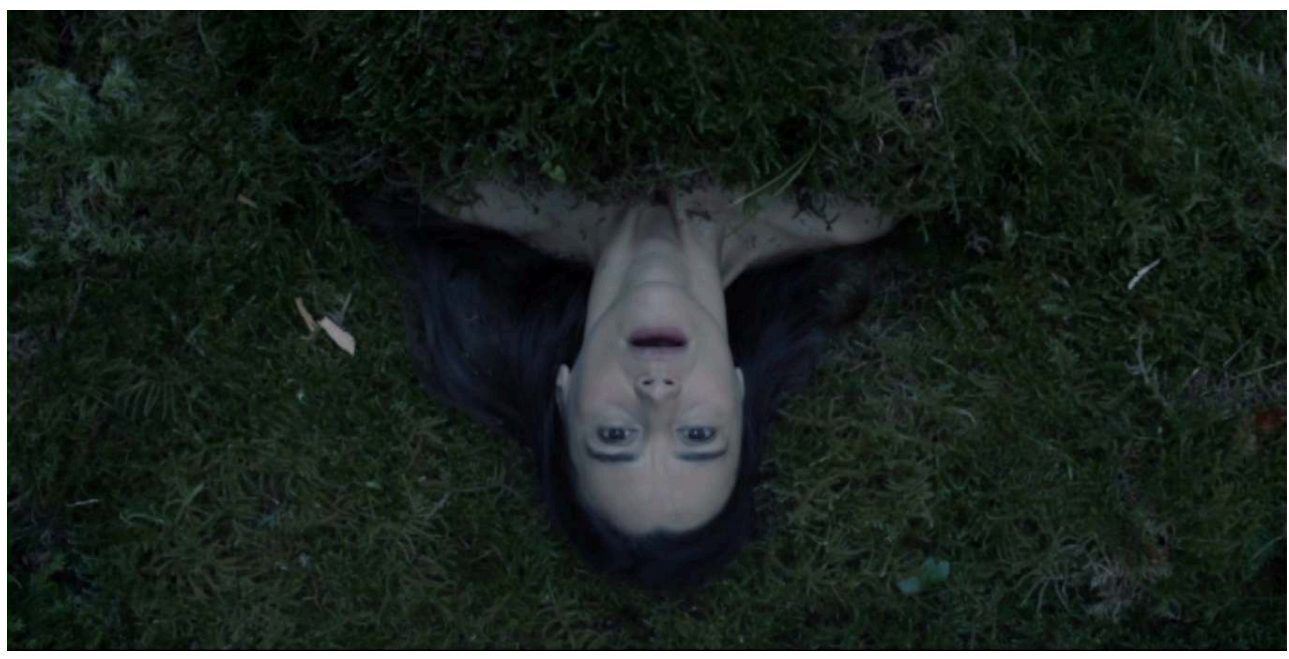

La fin de Zone blanche s'empare, à sa manière, d'une autre distinction chère à Deleuze, celle qu'il emprunte cette fois-ci à Antonioni et qui contraste le «petit horizon » de l'imaginaire cinématographique (et j'ajoute ici télévisuel) européen et «l'horizon cosmique » qui définit la science-fiction américaine. Cet « horizon qui n'en finit pas de reculer et de livrer ses monstres » nous invite à reconsidérer la fonction du paysage dans ces deux séries, ainsi que la relation qu'il présuppose entre l'humain et le nonhumain ${ }^{24}$. La pulsion bestiale qui motive les nombreux meurtres, amputations et défigurations commis tout au long de la première saison de Zone blanche affirme toujours plus l'emprise de cette forêt sur le milieu réaliste structurant le récit policier. A la fin de la saison, la forêt se trouve, par conséquent, redéfinie : elle est devenue ce monde originaire que Deleuze situe dans l'entre-deux, entre l'espace quelconque et le milieu déterminé, entre l'affect et l'action ${ }^{25}$.

Le monde originaire n'oppose donc pas la Nature aux constructions de l'homme : il ignore cette distinction qui ne vaut que dans les milieux dérivés. Mais surgissant entre un milieu qui n'en finit pas de mourir, et un autre qui n'arrive pas à naître, il s'approprie aussi bien les restes de l'un que les ébauches de l'autre pour en faire des 'symptômes morbides', comme dit Gramsci' ${ }^{26}$.

C'est dans la deuxième saison que cette tension morbide dont se nourrit Cernunnos commence à s'exprimer pleinement.

Plus généralement, les mondes dans lesquels Les Revenants et Zone Blanche nous plongent actualisent, de différentes manières, ces traits du monde originaire. Monde de pulsions, l'humain et l'animal ne cessent de s'y confondre. La violence et la cruauté surgissent beaucoup plus visiblement dans le milieu de Villefranche. Les Revenants efface au premier abord la monstruosité du mort-vivant, insistant sur l'apparence ordinaire de celui-ci, contrairement au grotesque gore et excessif des zombies de The Walking Dead ou de George Romero, par exemple. A travers ses deux saisons, Les Revenants affirme, cependant, une temporalité plus évasive qui enferme les personnages dans une destinée où « commencement » et « fin de monde » se brouillent. "C'est (le monde originaire) qui entraîne le milieu, » nous dit Deleuze, « et aussi qui en fait un milieu fermé, absolument clos, ou bien qui l'entrouvre sur un espoir incertain ${ }^{27}$ »- comme celui que suggère la filiation choisie de Julie et Victor, enfin réunis et libres dans l'espace vide de cette plage. Il ajoute :

[Celui-ci] n'existe et n'opère qu'au fond d'un milieu réel, et ne vaut que par son immanence à ce milieu dont il révèle la violence et la cruauté ; mais aussi le milieu ne se présente comme réel que dans son immanence au monde originaire, il a le 
statut d'un milieu 'dérivé' qui reçoit du monde originaire une temporalité comme $\operatorname{destin}^{28}$.

Dans Les Revenants et Zone Blanche, l'horizon cosmique, peuplé de monstres, semble ainsi continuellement contenu et refoulé au plus profond du petit horizon familial, local et national autour duquel s'organisent les séries. Dans un article récent, Claire Mouflard voit dans la mobilisation de ces processus de dégradation organique une critique des dérives anti-humanistes de la société européenne contemporaine, notamment dans la gestion des crises humanitaires qui ont accompagné l'arrivée massive de migrants depuis le début du $21^{\text {ème }}$ siècle ${ }^{29}$. Comme je l'ai démontré, espace, territorialité et écologie s'entremêlent intimement dans ces deux séries. L'écologie symbiotique caractéristique du monde originaire que décrit Deleuze s'y matérialise, en particulier, dans le mystère hydraulique et la liquidité mystique des corps des revenants ainsi que dans l'insertion de Laurène Weiss et de la caméra dans l'humus de la forêt à la fin de la première saison de Zone blanche. La lecture que j'ai proposée ici invite donc à percevoir dans les angoisses sociales, politiques et écologiques confrontées dans ces deux séries des préoccupations, plus latentes, de territorialité et de créativité culturelles.

Fig. 12. Vue depuis la grotte où Laurène Weiss a été tenue captive quand elle avait 18 ans. Zone blanche (S. 1, Ep. 1, 2017)

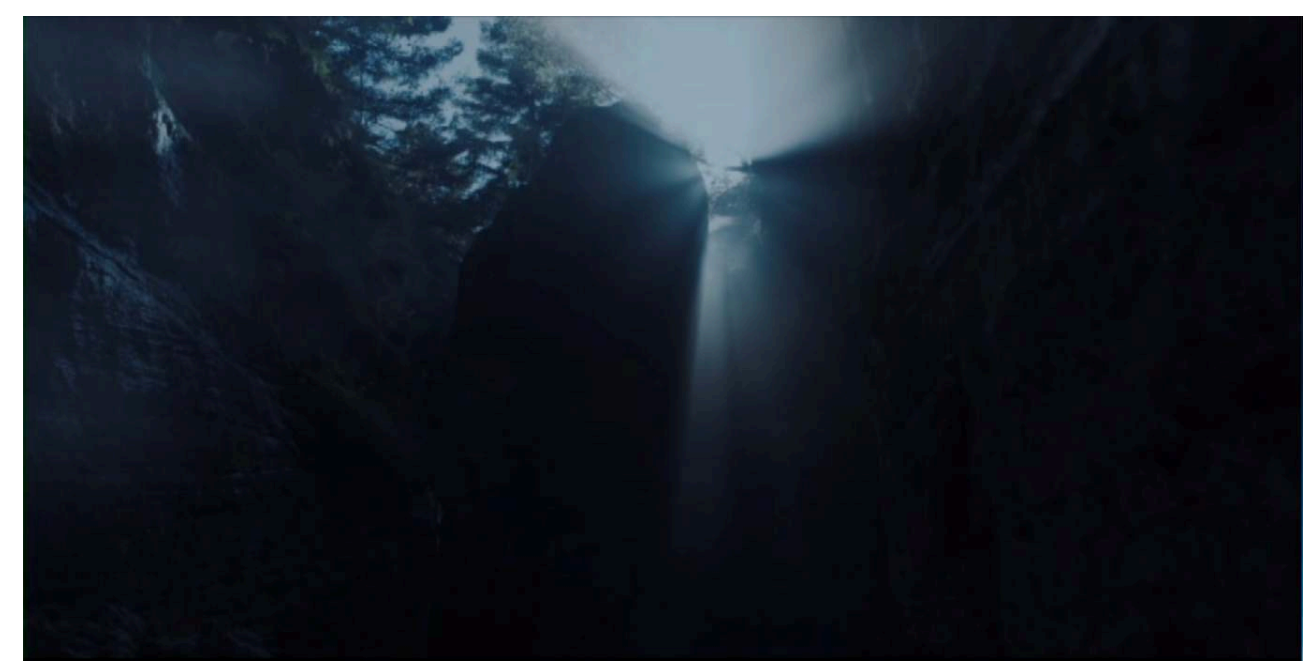

\section{BIBLIOGRAPHIE}

« Gilles Deleuze - Vérité et temps cours 53 du 24/01/1984 - 3 transcription : Julien Quélennec », La voix de Gilles Deleuze en ligne, Université Paris 8, Vincennes-Saint-Denis, http://www2.univparis8.fr/deleuze/article.php3?id_article=324.

AGGER, Gunhild, «The Killing: Urban topographies of a Crime », The Journal of Popular Television Vol. 1, No. 2, 2013, p. 235-242. 
BASIRICO, Benoît, « Interview Zone Blanche / Thomas Couzinier \& Frédéric Kooshmanian : `De la mélodie à des endroits précis plutôt que de l'ambiance partout' ", Cinezik.fr, 11 mai 2017, https:// www.cinezik.org/infos/affinfo.php?titre0=20170511225902.

CASTA, Isabelle Rachel, « Barrages dans la montagne... : le paysage des Morts dans Les Revenants », Entrelacs No. 4, 2016, 6 février 2018, http://journals.openedition.org/entrelacs/2160.

CÉLESTIN, Roger, « Lost in Globalized Space? A Certain French Cinema Abroad, » Yale French Studies, No. 115, New Spaces for French and Francophone Cinema, 2009, p. 31-46.

CLARK, Catherine E., JACOBSON, Brian R., « Les Revenants, Tignes, and the Return of Post-war Modernisation ", in France in Flux : Space, territory and Contemporary Culture, éd. Ari J. Blatt et Edward Welch, Oxford, Oxford University Press, 2019, p. 141-160.

CORTADE Ludovic, Le cinéma de l'immobilité, Paris, Éditions de La Sorbonne, 2008.

COULTHARD, Lisa, HORECK, Tanya, KLINGER, Barbara, MCHUGH, Kathleen, « Broken Bodies/Inquiring Minds: Women in Contemporary Transnational TV Crime Drama », Television \& New media, Vol. 19, No. 6, 2018, p. 507-514.

CREEBER, Glen, « Killing Us Softly: Investigating the Aesthetics, Philosophy and Influence of Nordic Noir Television, » Journal of Popular Television Vol. 3, No. 1, 2015, p. 21-36.

DELEUZE Gilles, L'image-mouvement, Paris, Éditions de Minuit, 1983.

DELEUZE Gilles, L'image-temps, Paris, Éditions de Minuit, 1985.

FERNANDEZ, Julia, « Zone Blanche - Mathieu Missoffe : 'J'avais envie de plus de codes d'aventure en saison 2, donc moins de polar' ", Allociné.fr, 18 février 2019, http://www.allocine.fr/article/ fichearticle_gen_carticle $=18679037 . \mathrm{html}$.

FOULATIER, Julien, « Gregory Crewdson : Entre la ville et la mort », Boum !Bang !, 4 mars 2013, http://www.boumbang.com/gregory-crewdson.

LANGLAIS, Pierre, «Un générique à la loupe : Les Revenants, nature morte », Télérama, 12 février 2019, http://www.telerama.fr/series-tv/un-generique-a-la-loupe-les-revenants-nature-morte, 90165.php.

MOREL, Josué, «Les Revenants : de l'inexistence des séries françaises », Cinechdoche, décembre 2012, http://cinecdoche.com/les-revenants-de-linexistence-des-series-françaises/, lien inactif.

MOUFLARD, Claire, « Zombies and Refugees: Variations on the 'Post-Human' and the 'Non-Human' in Robin Campillo's Les Revenants (2004) and Fabrice Gobert's Les Revenants (2012-2015) », Humanities Vol. 5, No. 48, numéro spécial Race, Politics, and the Humanities in an Age of 'Posts', 2016, p. 2-11.

POITTE, Isabelle, «Zone Blanche, saison 2 : 'La mythologie passe au premier plan' », Télérama, 11 février 2019, https://www.telerama.fr/series-tv/zone-blanche,-saison-2-la-mythologie-passe-aupremier-plan,n6118400.php.

TALLY JR., Robert T., Topophrenia : Place, Narrative, and the Spatial Imagination, Bloomington, Indiana University Press, 2018.

\section{NOTES}

1. Le site IMDB (https://www.imdb.com/name/nm3197544/) nous révèle qu'il a participé, entre autres, à l'écriture de plusieurs épisodes des séries Profilage (2009-2014) et Engrenages (2005-2019) 
ainsi qu'à celle de plusieurs téléfilms de la série "Mystère à Paris »-Mystère au Moulin Rouge (2011), Mystère à la Tour Eiffel (2015), et Mystère à l'Opéra (2015)-diffusée sur France 2 (consulté le 18 octobre 2019).

2. Netflix a en effet repris les droits de la série en 2019 (la première saison était disponible en 2018 sur Amazon Video prime), et les deux saisons sont accessibles au public américain depuis fin juin 2019.

3. Roger Célestin, "Lost in Globalized Space? A Certain French Cinema Abroad ", Yale French Studies, No. 115, «New Spaces for French and Francophone Cinema », 2009, p. 39 [p. 31-46]. Célestin interroge ici, en traduisant ses propos en français, la capacité de la France à produire et exporter, avec constance, des films pouvant rencontrer un succès commercial qui ne soient ni 'Hollywoodiens' ni des films qui recycleraient une « certaine idée de la France ».

4. Célestin, p. 45.

5. Isabelle Rachel Casta, «Barrages dans la montagne...: le paysage des Morts dans Les Revenants ", Entrelacs No. 4, 2016, http://journals.openedition.org/entrelacs/2160, consulté le 4 mai 2019.

6. Josué Morel, «Les Revenants, de l'inexistence des séries françaises », Cinecdoche, décembre 2013, http://cinecdoche.com/les-revenants-de-linexistence-des-series-françaises/, lien inactif.

7. Isabelle Rachel Casta, op. cit.

8. Robert T. Tally Jr., Topophrenia: Place, Narrative, and the Spatial Imagination, Bloomington, Indiana University Press, 2018, p. 23. La topophrénie, selon Tally, caractérise ainsi l'engagement subjectif, mais maladif, qu'un sujet développe avec un lieu donné. Ce positionnement pathologique l'amène ainsi à y envisager l'existence d'espaces parallèles possibles.

9. Catherine E. Clark et Brian R. Jacobson se sont récemment intéressés à la série dans son évocation du barrage de Tignes et de la mise en œuvre de gros projets de modernisation étatiques dans les Alpes à partir des années 1950. Cette analyse a donné lieu à un chapitre, «Les Revenants, Tignes, and the Return of Post-war Modernisation », publié dans France in Flux : Space, territory and Contemporary Culture, éd. Ari J. Blatt et Edward Welch, Oxford, Oxford University Press, 2019, p. 141-160.

10. Benoit Basirico, «Interview Zone Blanche/Thomas Couzinier \& Frédéric Kooshmanian, 'De la mélodie à des endroits précis plutôt que de l'ambiance partout' », Cinezik.fr, 11 mai 2017,

https://www.cinezik.org/infos/affinfo.php?titre0=20170511225902, consulté le 22 octobre 2019.

11. Lisa Coulthard, Tanya Horeck, Barbara Klinger et Kathleen McHugh, ont récemment publié un numéro spécial sur le sujet, «Broken Bodies/Inquiring Minds : Women in Contemporary Transnational TV Crime Drama ", dans la revue Television \& New media, Vol. 19, No. 6, 2018, p. 507-514.

12. Propos recueillis par Julia Fernandez, «Zone Blanche - Mathieu Missoffe : 'J'avais envie de plus de codes d'aventure en saison 2, donc moins de polar'», Allociné, 18 février 2019, http:// www.allocine.fr/article/fichearticle_gen_carticle=18679037.html, consulté le 22 octobre 2019.

13. Glen Creeber, «Killing Us Softly: Investigating the Aesthetics, Philosophy and Influence of Nordic Noir Television ", Journal of Popular Television, Vol. 3, No. 1, 2015, p. 22 [p. 21-36].

14. Gunhild Agger, «The Killing: Urban topographies of a Crime », The Journal of Popular Television Vol. 1, No. 2, 2013, p. 235-242.

15. Entretien avec Mathieu Misoffe par Isabelle Poitte, «Zone Blanche, saison 2 : 'La mythologie passe au premier plan' ", Télérama, 11 février 2019, https://www.telerama.fr/series-tv/zoneblanche,-saison-2-la-mythologie-passe-au-premier-plan,n6118400.php, consulté le 28 avril 2019.

16. Gilles Deleuze, L'Image-Mouvement, Paris, Éditions de Minuit, 1983, p. 154-155.

17. Pierre Langlais, « Un générique à la loupe : Les Revenants, nature morte », Télérama, 12 février 2019, http://www.telerama.fr/series-tv/un-generique-a-la-loupe-les-revenants-nature-morte, 90165.php, consulté le 22 octobre 2019.

18. Gilles Deleuze, L'Image-Temps, Paris, Éditions de Minuit, 1985, p. 27. 
19. Ibid.

20. Julien Foulatier, "Gregory Crewdson : Entre la ville et la mort », Boum!Bang!, 4 mars 2013, https://www.boumbang.com/gregory-crewdson/, consulté le 4 mai 2019.

21. Ludovic Cortade, Le cinéma de l'immobilité, Paris, Éditions de La Sorbonne, 2008, p. 101.

22. Cortade, p. 86.

23. Gilles Deleuze, L'image-temps, p. 34.

24. «Gilles Deleuze - Vérité et temps, cours 53 du 24/01/1984 - 3 transcription: Julien Quélennec ", La voix de Gilles Deleuze en ligne, Université Paris 8, Vincennes-Saint-Denis, http:// www2.univ-paris8.fr/deleuze/article.php3?id_article=324, consulté le 29 avril 2019.

25. Deleuze, L'image-temps, p. 173-174.

26. Deleuze, L'image-mouvement, p. 195.

27. Deleuze, L'image-mouvement, p. 176-177.

28. Deleuze, L'image-mouvement, p. 175.

29. Claire Mouflard, "Zombies and Refugees: Variations on the 'Post-Human' and the 'NonHuman' in Robin Campillo's Les Revenants (2004) and Fabrice Gobert's Les Revenants (2012-2015) », Humanities, Vol. 5, No. 3, numéro spécial Race, Politics, and the Humanities in an Age of 'Posts', 2016, p. $2-11$.

\section{RÉSUMÉS}

Cet article examine la mise en scène de plus en plus commune dans les séries télévisées policières et/ou fantastiques francophones (principalement françaises et franco-belges) de territorialités composites et topographies mobiles. M'appuyant principalement sur Les Revenants (Canal+, 2012-2015) et Zone blanche (France 2, 2017), je m'intéresse en particulier à ce que je vois comme une double impulsion paradoxale dans le travail des scénaristes et réalisateurs francophones depuis quelques années. Inspirés notamment par l'engouement international pour l'esthétique sérielle scandinave, ces derniers mettent l'accent sur une temporalité plus longue et plus lente de la progression de l'intrigue et de sa résolution. D'une part, ces séries opèrent une déterritorialisation visible $d u$ récit. Bien que celui-ci se déroule systématiquement dans une communauté rurale ou du moins étrangement isolée, l'espace échappe à toute localisation spécifique et se définit plutôt par son caractère dénationalisé, assemblage d'éléments hétéroclites et marqueurs géographiques divers. D'autre part, et c'est là qu'émerge le paradoxe, ces séries revendiquent la centralité narrative et esthétique d'un enracinement géographique et écologique double. Affirmant un lien inextricable entre l'humain et le naturel, ces deux séries mobilisent des récits historiques et mythologiques locaux spécifiques. Si tout effort de différencier espaces local, national et global s'y trouve ainsi déstabilisé, le paysage, lui, s'y voit réactivé et animé là où, traditionnellement, il était réduit à sa simple présence spectaculaire et son affirmation d'une identité régionale ou nationale. Le monde que nous découvrons dans ses diverses manifestations atmosphériques, écologiques et géologiques, se transforme en un site/ lieu défini autant par son immobilisme que par sa mobilité, signifiant, comme le proposait Gilles Deleuze dans son livre L'Image-Temps (1985), à la fois l'absence et la présence, le vide et le plein, la vie et la mort. Si l'intertextualité référentielle déployée à travers ces séries suggère une appropriation, et une transposition, de codes génériques étrangers, je propose que nous y voyions plutôt la mise en scène et le questionnement de ce rapport. 
This article examines the recurrence in Francophone crime and/or fantastic TV dramas (mainly French and Franco-Belgian) of multifarious territorialities and mobile topographies. Focusing primarily on Les Revenants/The Returned (Canal+, 2012-2015) and Zone blanche/Black Spot (France 2, 2017), I consider a twofold, but paradoxical, impulse in the work of Francophone screenwriters and TV directors in the last few years. Inspired by the growing international popularity of Scandinavian serial aesthetics, Francophone televisual screenwriting has placed more emphasis upon extended temporalities, slow-pace narratives, and delayed plot resolutions. Narratives also reveal visible efforts to destabilize geographical inscriptions within easily recognizable spaces. On the one hand, these series systematically take place in communities that are either rural or, at the very least, strangely isolated. Space, however, is de-territorialized, defined by an assemblage of heterogeneous elements and varied geographical markers. On the other hand, somewhat paradoxically, the local ecological environment firmly takes the central stage in the aesthetic and narrative singularity of the series. The human and the natural worlds are intimately connected, while the plot draws from specific local historical and mythological references. Distinctions between the local, the regional, and the national are blurred. Most importantly, landscapes no longer function as a mere background spectacle or distinctive regional and national identity markers. They are, in contrast, reactivated and animated, with greater emphasis placed upon their atmospheric, ecological, and geological qualities. As a result, the worlds they reveal become sites/sights that, following Gilles Deleuze's analysis in L'Image-Temps/ Cinema 2: The Time-Image (1985), are poised between immobility and mobility, absence and presence, emptiness and plenitude, and, ultimately, life and death. This article, therefore, argues that the intertextuality at play across these two series achieves more than easy appropriations and transpositions of international genres; it calls into question the current status of Francophone television drama and its relationship to international productions.

\section{INDEX}

Mots-clés : Revenants (Les), Zone blanche, Deleuze Gilles, topographies mobiles, paysage, espace Keywords : Returned (The), Black Spot, Deleuze Gilles, mobile topographies, landscapes, space

\section{AUTEUR}

\section{AUDREY EVRARD}

Audrey Evrard enseigne dans le département de Langues et Littératures Modernes à l'Université Fordham à New York, aux États-Unis. Ses recherches se concentrent depuis plusieurs années sur le cinéma français, notamment le documentaire sur lequel elle a publié plusieurs articles dans diverses revues anglophones, telles que Jump Cut: A Review of Contemporary Media, Modern and Contemporary France, Working USA: The Journal of Labor and Society, Nottingham French Studies, Contemporary French Civilization.

Audrey Evrard is Assistant Professor of French in the Department of Modern Languages and Literatures at Fordham University in New York City, USA. Specializing in French film studies, she has published articles on documentary filmmaking, a focus of her research, in various anglophone journals, including Jump Cut: A Review of Contemporary Media, Modern and Contemporary France, Working USA: The Journal of Labor and Society, Nottingham French Studies, Contemporary French Civilization. 